\title{
Radioactive Decay as A Second-Order Kinetics Transformation Process. Consequences on Radiometric Dating
}

\author{
Giancarlo Cavazzini ${ }^{1}$ \\ ${ }^{1}$ CNR Istituto di Geoscienze e Georisorse, Padova, Italy \\ Correspondence: Giancarlo Cavazzini, CNR Istituto di Geoscienze e Georisorse, Padova, Italy. Tel: \\ 0039-049-827-9137. E-mail: giancarlo.cavazzini@igg.cnr.it
}

Received: November 14, 2019

Accepted: November 30, 2019

Online Published: December 3, 2019

doi:10.5539/apr.v12n1p26

URL: http://dx.doi.org/10.5539/apr.v12n1p26

\begin{abstract}
Arguments suggest and recent analysis of experimental work confirm that the current interpretation of the transformation process we call 'radioactive decay' should be revised. The characteristics of this process are better accounted for by re-interpreting it in terms of second-order kinetics. Therefore, the atomic systems of nuclides we observe decay are 'radio-activated', and not, as hitherto believed, 'radio-active'. According to this interpretation, the rate of decay of a radioactive nuclide is at any instant proportional to the concentration of the physical species that determines its activation. The analysis of $\lambda$ of alfa- and beta-emitting nuclides show the dependence of these parameters from solar activity and distance. Therefore, if changes in the emission of energy from the sun occurred over time since the formation of a geological system, changes in the values of $\lambda$ of the radioactive nuclides would also have occurred, and the calculated radiometric age of the system may differ from the true age. Implications on the science of dating geological samples using parent-daughter decay systematics are investigated.
\end{abstract}

Keywords: Radioactive decay, Second-order kinetics, Decay constant, Solar activity, Apparent age

\section{Introduction}

Recent careful analysis of measurements of activity of beta-emitting $\left({ }^{32} \mathrm{Si},{ }^{36} \mathrm{Cl}\right.$, ) alpha-emitting $\left({ }^{226} \mathrm{Ra}\right)$, and electron-capturing $\left({ }^{54} \mathrm{Mn}\right)$ nuclides have shown that variations of the respective decay constants occur in relation to sun activity and distance ${ }^{1-7}$ (Fischbach et al., 2009; Jenkins \& Fischbach, 2009; Jenkins et al., 2009; 2010; 2102; Javorsek II et al., 2010; Sturrock et al., 2011).

A clear periodic oscillation with an approximate period of one year has been observed for the rate of decay of ${ }^{36} \mathrm{Cl}$ for seven successive years (from July 2005 to June 2011), during instrumental calibration at The Ohio State University Research Reactor (OSURR) (Jenkins et al., 2012), and similar oscillations also result from the analysis of half-life measurements taken by two other independent groups: at the Brookhaven National Laboratory (BNL), New York, and at the Physikalisch-Technische Bundesanstalt (PTB) in Braunschweig, Germany.

The BNL group measured the half-life of ${ }^{32} \mathrm{Si}$ for four years (1982-1986), and the PTB group measured the half-life of ${ }^{226} \mathrm{Ra}$ in a standard used for comparison in the measurements of the half-life of ${ }^{152} \mathrm{Eu}$ for fifteen years (1984-1998) (Jenkins et al., 2009; the raw data are from Alburger et al., 1986, and Siegert et al., 1998, respectively). The counting rates of each of these nuclides increase to a maximum in winter, and progressively decrease to a minimum in summer, suggesting a direct relationship between half-lives of nuclides on the Earth and Earth-Sun distance.

In the analysis of the raw data from BNL and PTB, the data sets on ${ }^{32} \mathrm{Si}$ and ${ }^{226} \mathrm{Ra}$ were shown to have not only the same period but, in the two years during which the two data sets overlapped, they also had the same approximate phase and amplitude (Jenkins et al., 2009; Javorsek II et al., 2010). Moreover, the hypothesis of a solar influence on radioactive decays was strengthened by the identification of an additional periodicity in the BNL data at 11.25/year and in the PTB data at 11.21/year. Both of these peaks can be related to the rotation (and probably to the inhomogeneous nature) of the core of the Sun (Sturrock, 2009).

A third periodicity was also identified by Sturrock et al. (2011) in both the BNL and PTB data sets, which is analogous to Rieger periodicity (Rieger et al., 1984). 
Jenkins and Fischbach (2009), Jenkins et al. (2009) and Fischbach et al. (2009) also report a relationship between solar activity and the measured value of the decay rate of ${ }^{54} \mathrm{Mn}$, during a series of solar flares from the beginning of December 2006 to the beginning of January 2007. During some flares, the value of the decay constant of ${ }^{54} \mathrm{Mn}$ strikingly changed by a factor of $\sim 2$. As correctly suggested by Fishbach et al. (2009), all these observations require a revision of the current model used to describe the process of radioactivity.

Long before these interesting observations, arguments, which we will illustrate in the following, suggested that a revision of the model which describes the process of radioactive decay was needed. The characteristics of this process of transformation are more properly accounted for if re-interpreted in terms of second-order kinetics. Moreover, the relationship between the change in solar activity and the change in the rate of decay of ${ }^{54} \mathrm{Mn}$ raises the question whether the decay constants of the radioactive nuclides were actually constant during the geologic time. For this reason, we will also discuss implications that a second-order kinetics re-interpretation has on the science of dating geological systems using parent-daughter decay systematics. A slow decrease in sun activity over time-spans of the order of $10^{9}$ years may have produced changes in the value of the decay constants which cannot be neglected.

\section{On the Way to Ascertain if a System is Stable/unstable, and on the Inconsistency of the Exponential Relationship Used to Quantitatively Describe the Decay of the Radioactive Nuclides with an Internal (self-) Transformation Process}

\subsection{Stability and Instability}

Let us start our critical analysis of the current interpretation of the process of radioactive decay by briefly discussing the method to ascertain if a system is stable or not. First of all, we have to define precisely what we mean by 'unstable': 'unstable' is a system which transforms itself 'spontaneously', i.e. a system which has within itself the cause of its own instability.

We call a system 'unstable' if we observe values of its properties changing over time. We have no other way to ascertain the instability/stability of a system. Obviously, one could correctly argue how long the time-span of observation of a system should be before the stability/instability of the system is ascertained. As a matter of fact, there is not and there cannot be at all any a priori time-span of observation: the system could modify very slowly, so that the longer the time-span of observation, the higher our confidence in believing the system stable; and, on principle, we have no choice but the continuous observation of the properties of the system over an infinite time-span to ascertain if the system is actually a stable one. Thus, we define 'stable' a system whose proprieties change after an infinite time-span of observation.

However, it is obvious that observing a system for an infinite time-span would hinder any decision about the system itself. Thus, we usually call 'stable' a system whose properties do not change over a time-span which is a reasonable (and often quite small) fraction of our biological life-time. If this is the rule, it seems very improper calling unstable the atoms of nuclides such as ${ }^{238} \mathrm{U}$ and ${ }^{232} \mathrm{Th}$, which were in the solar nebula long before their incorporation in the planet we live, the formation of which is believed to have occurred approximately 4.5 billion years ago.

\subsection{Internal Instability}

In spite of this simple line of reasoning, the transformation process of atoms of nuclides we called 'radio-active' was believed to be due to internal reasons, not related to interactions of these systems with something in their surroundings. This was essentially due to two reasons. The first reason was that experiments showed that, although the atoms of the radioactive nuclides are certainly non-isolated systems and therefore interacting with their surroundings, their rate of transformation did not appreciably change as the external conditions changed.

Fischbach et al. (2009) correctly emphasized that few issues frame the history of natural radioactivity as fundamentally as the question of whether the decay rates of nuclides are constants of nature, unaffected by the external environment. After Henri Becquerel discovered radioactivity, in 1896, intense efforts were made to ascertain whether the decay rates of nuclides could be affected by temperature, pressure, chemical composition, electric and magnetic fields etc., and Rutherford et al. (1930) concluded that the rate of transformation of a radioactive nuclide is a constant under all conditions.

The second reason was that the results of the measurements of the rate of transformation of these atomic systems can be interpreted as if the process occurred according to first-order kinetics, i.e. in terms of probability to decay (Rutherford et al., 1930). According to this interpretation, all the atoms of a radioactive nuclide have equivalent probability to decay in a certain time-span of observation. However, some of them decay whereas the others do not, 
and the parameter we call the "decay constant" of the transformation, denoted by the Greek letter $\lambda$, represents the instantaneous probability one atom has to transform itself in a unit of time:

$$
-\frac{d N}{N} \frac{1}{d t}=\lambda
$$

All the atoms in a number of atoms of a radioactive nuclide have the same probability to decay in a time-span of observation because they are equivalent. However, if the atoms are equivalent and the transformation of each of these atomic systems is due to purely internal reasons, why some of them transform and some others do not? Why should the behaviour of systems which are equivalent, and which transform themselves only due to internal reasons, be different?

\subsection{The Exponential Law of Decay is Inconsistent with Internal Instability}

Finally, let us consider the decrease over time in the number of atoms of radioactive nuclides. The law of decrease is the exponential law which results from integration of differential equation (1):

$$
N=N_{0} e^{-\lambda t}
$$

where $N_{0}$ is the number of atoms at $t=0$.

According to this equation, the number of atoms of the radioactive nuclide, $N$, will never be zero, unless an infinite time-span has passed. This is paradoxical, indeed, because, according to the line of reasoning we have illustrated in 2.1 , this is absolutely inconsistent with the concept of instability of (some of) the atomic systems, a fortiori with the concept of internal instability. Therefore, relationship (2) cannot describe the change over time in a number of self-unstable systems, and two are the possible reasons for this inconsistency: i) the process of transformation of the atoms of the radioactive nuclides is not due to purely internal reasons; alternatively, ii) relationship (2) is wrong. However, relationship (2) is the result of repeated accurate experimental work on all radioactive species, and we can exclude it is wrong. Therefore, we infer that the processes of decay are not the result of processes of self-transformation, but they result from interactions of atomic systems with something external, as also suggested by lines of reasoning in 2.1 and 2.2: the atomic systems we currently call 'radio-active' are, instead, radio-activated.

"Activation" is not new in physics: processes such as induced nuclear fission and neutron-activation are examples. The latter is a well-known analytical method which is based on transformations of nuclides due to interaction with neutrons. Therefore, there is no conceptual difference between processes as nuclear fission or neutron activation and the process of radioactive decay. The difference is that we know that physical entities we call 'neutrons', by interaction with atoms of some nuclides, cause their fission or transformation, whereas we do not know (because we do not 'see' them) the physical entities which, by interaction with atoms of some nuclides, cause their 'radioactive' transformation.

That is one reason why scholars who investigated this physical process believed that the transformation of these atomic systems was due to internal instability. However, the law of decay eqation (2) they calculated showed that this hypothesis was incorrect, indicating that the concept of probability to decay had to be substituted by the concept of probability to interact with some external physical species.

\section{Radioactive Decay as a Second-order Kinetics Transformation Process}

Relationship (2) can properly describe the decrease over time in the number of systems which transform by interaction with some external entities of matter/energy, i.e. according to second-order kinetics.

In chemistry, second-order kinetics is the kinetic relationship of transformation of molecules of a chemical species $X$ for interaction with a chemical species $Y . X$ and $Y$ are called the 'reactants':

$$
\frac{d[X]}{d t} \propto-[X][\mathrm{Y}],
$$

where $d[X] / d t$ is the instantaneous rate of decrease of the concentration of species $X,[X]$, and $[Y]$ is the concentration of species $Y$ at the same instant.

Nevertheless, as discussed in 2.2, the results of the measurements of activities over time led to interpreting the process in terms of probability of self-transformation, a process which occurs according to first-order kinetics. Therefore, we also write: 


$$
\frac{d[X]}{d t}=-\lambda[X]
$$

In order that equations (3) and (4) are simultaneously valid it is necessary that the concentration of the reactant $Y$ is constant over the time-span the measurements of activity are carried out:

$$
\lambda \propto[Y]
$$

If this is the case, a process which occurs according to second-order kinetics may be mistaken for a process which occurs according to first-order kinetics; and the value of the decay constant of the transformation contains information on the value of the concentration of reactant $Y$.

We hypothesize that the value of $\lambda$ is approximately constant during measurements of activity because the value of the concentration of species $Y$ is approximately constant. If this is correct, how can the concentration of species $Y$ be constant over time?

If we assume that each element of species $Y$ is 'consumed' when activating one atom of species $\mathrm{X}$, a simple explanation to this question is that species $Y$ is immediately and continuously reintroduced in the system as it 'disappears' by interaction with the atoms of the nuclide which is being activated. In this case, the concentration of species $Y$ in a certain volume of matter remains substantially constant over time. This condition is realized if a continuous 'flux' of elements of $Y$ exists, which pervades the matter. This flux must be approximately stable over time-spans which are important fractions of the human biological life, because repeated measurements of activities of nuclides over 30-50 years did not give results which were clearly different.

The process of transformation can be described as follows: the atoms of a radioactive nuclide are targets which may be hit or not by elements of the physical species $Y$ which can determine their activation. In a very small time-span $d t$ (an 'instant'), the number of the targets which are hit in the unit of time and which decay to the daughter species are obviously proportional to the total number of targets and to the concentration of species $Y$ at that instant:

$$
\frac{d N}{d t} \propto-[Y] N
$$

Since the rate of transformation depends on the number of targets, the number $N$ of atomic systems which can be activated in a unit of time decreases with time and tends to zero, without ever reaching it, as correctly shown by the exponential relationship (2).

We assume that all the atomic systems of a nuclide species $X$ are equivalent from the point of view of the content in energy. We also assume that the elements of $Y$ are characterized by a continuous spectrum of values of energy in the range $E_{1} \div E_{2}\left(E_{2}>E_{1}\right)$, and that only the elements which are characterized by a content in energy which is higher than a certain threshold value $\varepsilon_{0}$ may determine the decay of $X$. Accordingly, we write the rate of decay of nuclide $X$ as:

$$
\frac{d N}{d t}=-\varphi[Y] N=-\frac{\int_{\varepsilon_{0}}^{E_{2}} v d E}{\int_{E_{1}}^{E_{2}} v d E}[Y] N
$$

where $v$ is the fraction of the elements of $Y$ as a function of energy, and the ratio between the two integrals represents the fraction $\varphi$ of $Y$ which can determine the activation of nuclide $X$.

At present, the remarkable fact is that accurate measurements have shown that the decay 'constants' of radioactive nuclides depend on solar 'activity' and distance (Jenkins \& Fischbach, 2009; Fischbach et al., 2009; Javorsek II et al., 2010; Sturrock et al., 2011; Jenkins et al., 2009, 2010, 2012). This could mean that the physical species which causes the activation of the nuclide systems are originated in the sun or, alternatively, that the decay process represents the result of an effect of the sun on physical entities which may not strictly originate in the sun, transforming these entities into activators. As regards the nature of these physical entities and of their flux, at the present stage of knowledge any hypothesis is rather speculative, even if neutrinos from the sun have been proposed to play a role (Fischbach et al., 2009). 
Whatever the case, this fact has the following important consequence: in space and time the same radioactive nuclides in planetary and sub-planetary matter orbiting around the same star or around different stars generally decay according to different rates. This has implications on the meaning of the age values we calculate from parent-daughter nuclide systematics to study the history and evolution of that planetary matter, which we discuss in the following.

\section{Change over Time of the Decay 'Constants' of Radioactive Nuclides in Planetary Matter Around a Star: Consequences on Calculated Radiometric Dates}

It is believed that stars undergo processes of evolution. An initial time-span, in which the production, and therefore the emission of energy increases over time (when the star begins to bright) is followed by a time-span in which the emission of energy is thought to be rather stable. After this time-span, the production/emission of energy progressively decreases to certain levels. The energy radiation per unit of time of a star which lies on the 'main sequence' (like our Sun), for a given chemical composition and stellar age, depends on its mass.

The observed relationship between activity in the sun and the value of the decay 'constant' of a nuclide raises the question whether the value of this parameter was actually constant during the stages of life of the sun and of the solar system. If the value of the decay constant of a radioactive nuclide depends on the concentration of the physical species that determines its activation, and if the concentration of the physical species which activates the nuclide changed over time, then the value of the decay 'constant' changed over time, and the value which we measure presently is only a point above a long variation curve.

This is an important question, because we calculate the age of terrestrial (and extra-terrestrial) geological events i.e. the formation of rock and mineral systems - by the well-known equation (in the simplified form):

$$
t_{a}=\frac{1}{\lambda} \ln \left(1+\frac{D}{N}\right),
$$

where $D, N$ represent, respectively, the number of atoms of daughter nuclide and parent nuclide at time $t$ after the formation of the system, and $\lambda$ is the value of the decay 'constant' we measure presently.

This shows that the calculated age $t_{a}$ may significantly differ from the true age $t$ due to hyperbolic dependence on the value of the 'constant', and this is the reason why we have added symbol $a$ to symbol $t$, indicating that the calculated age is an apparent one. If the value of $\lambda$ changed during the stages of life of the Sun, we calculate only apparent ages, and how much the apparent age deviates from the true age must be discussed.

\subsection{Linear Decrease of the Decosy 'Constant'}

If we assume the value of $\lambda$ to decrease over the true age $t$ of a geological system according to linear relationship

$$
\lambda=\lambda_{0}+m t
$$

where $\lambda_{0}$ is the value of the decay 'constant' of the nuclide at the time of formation of the geological system, $\lambda$ is the value of this parameter we measure presently, and $m$ is the angular coefficient of the evolution straight line. The true age of the geoòogical system is one of the two solutions (the positive one) of the following second-degree equation:

$$
-\frac{m}{2} t^{2}+\lambda t-\ln \left(1+\frac{D}{N}\right)=0 .
$$

Eq. (10) reduces to eq. (8) in case $m t^{2}$ is so small to be negligible. The true age $t$ is obtained by the equivalence between the value of $t$ in eq. (9) and the value of $t$ as solution of eq. (10), assuming the initial value $\lambda_{0}$ of the decay 'constant'. This calculation is articulated in three steps: first we assume $\lambda_{0}$ and calculate $m t=\lambda-\lambda_{0}$ (eq. (9)). Then we replace $m t$ into eq. (10) obtaining an equation of first degree in variable $t$. Finally, we solve this equation using the measured value of the ratio $D / N$.

According to this scheme of calculation, the true age of the system is given by the formula:

$$
t=\frac{\lambda}{m}\left\{1-\sqrt{1-\frac{2}{\lambda}\left(\frac{m}{\lambda}\right) \ln \left(1+\frac{D}{N}\right)}\right\},
$$

or

$$
t=\frac{2}{\lambda_{0}+\lambda} \ln \left(1+\frac{D}{N}\right) .
$$


For example, let us consider ${ }^{87} \mathrm{Rb}-{ }^{87} \mathrm{Sr}$ dating systematics, and consider $D / N$ such that eq. (8) gives $t_{a}=4.5 \mathrm{Ga}(D / N$ $\sim 0.06599$, since the present value of $\lambda^{87} \mathrm{Rb}$ is $1.42 \cdot 10^{-11} \mathrm{a}^{-1}$; Steiger and Jager, 1977). Assuming $\lambda_{0}=1.50 \cdot 10^{-11} \mathrm{a}^{-1}$ (a deviation of $5.6 \%$ from the present value, i.e. a change of $1.25 \cdot 10^{-9} \%$ per year), we calculate $m=-1.775 \cdot 10^{-22} \mathrm{a}^{-2}$ and $t=4.38 \mathrm{Ga}$. If we assume $\lambda_{0}=1.55 \cdot 10^{-11} \mathrm{a}^{-1}$ we calculate $m=-2.982 \cdot 10^{-22} \mathrm{a}^{-2}$ and $t$ approximately $4.30 \mathrm{Ga}$.

Therefore, the ratio $r$ between the true age (12) and the apparent age (8) has the same value for systems which have the same age (i.e. the same value of $\lambda_{0}$ ):

$$
r=\frac{2 \lambda}{\lambda_{0}+\lambda} .
$$

\subsection{Exponential Decrease of the Decay 'Constant'}

We can alternatively assume that the value of $\lambda$ decreased over time according to an exponential function. We write:

$$
\lambda=\lambda_{0} e^{-c t}
$$

where $\lambda_{0}$ is the value of the decay constant at the instant of formation of the system, and $c$ is a positive constant which represents the ratio between the rate of change with time of the concentration of the physical species that determines the activation of the parent nuclide and the concentration of this physical species at any instant.

In this case, differential equation (1) becomes:

$$
-\frac{d N}{N}=\lambda_{0} e^{-c t} d t
$$

which is integrated to:

$$
N=N_{0} \exp \left[\frac{\lambda}{c}\left(1-e^{c t}\right)\right]
$$

Eq. (16) correctly reduces to eq. (2) in the case the quantity $c t$ is small, so that the Mc Laurin's series expansion of the exponential within the argument of the exponential can be truncated to first-order term with small deviations from the exact value. This occurs a fortiori during laboratory measurements of activity of nuclides, because the time-span over which the measurements are carried out is small.

From eq. (16), we calculate the true age as:

$$
t=\frac{1}{c} \ln \left[1+\frac{c}{\lambda} \ln \left(1+\frac{D}{N}\right)\right]
$$

or

$$
t=-\frac{1}{c} \ln \left[1-\frac{c}{\lambda_{0}} \ln \left(1+\frac{D}{N}\right)\right] .
$$

\subsection{The Relationship between the True Age and the Apparent Age}

It is important to determine the relationship existing between the apparent age and the true age of the geological system. In the case of linear decreasing of $\lambda$, re-arranging of eq. (10) gives:

$$
t_{a}=t-\frac{m t^{2}}{2 \lambda},
$$

which shows that the difference between the apparent age and the true age is proportional to the square of the true age.

In the case of exponential decrease of $\lambda$, we calculate the relationship between the two age values by substituting eq. (8) into eq. (17) and re-arranging. We obtain:

$$
t_{a}=\frac{1}{c}\left(e^{c t}-1\right)
$$


The relationship between the apparent age and the true age depends on the values of the parameters $m / \lambda$ and $c$, respectively. In the case of linear decrease of $\lambda$, the apparent age and the true age approximately coincide if the ratio $m / \lambda$ is sufficiently small with respect to true age $t$. In the case of exponential decrease of $\lambda$, the two age values approximately coincide if parameter $c$ is sufficiently small with respect to true age $t$ so that $e^{c t} \sim 1-c t$.

In the other cases, the apparent age is higher than the true one - the apparent time and the true time running differently, the former running faster than the latter - and the deviation between the two age values increases as the true age increases. Since the relationships between the two age values are not linear, the deviation between the two age values does not increase linearly as the true age increases, i.e. the apparent time runs progressively faster than the true time as the true age increases (Fig. 1).

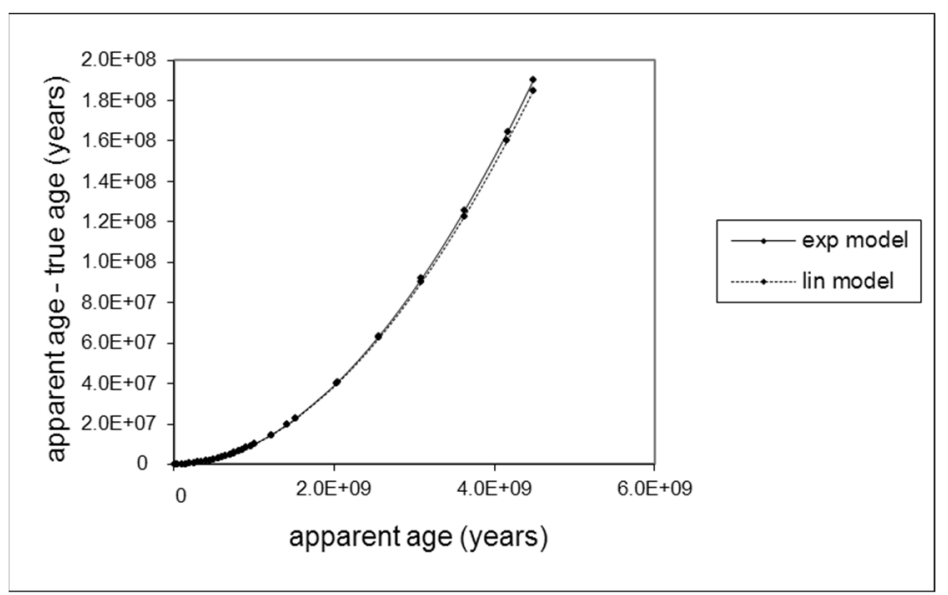

Figure 1. Difference between the apparent age and the true age of a geological system as a function of the apparent age in the case of exponential and linear models of decrease of $\lambda$. The two curves are calculated assuming $c=-m / \lambda$ $=2.1 \cdot 10^{-11} \mathrm{a}^{-1}$. See text for further explanation

Let us to refocus on the ${ }^{87} \mathrm{Rb}_{-}{ }^{87} \mathrm{Sr}$ dating systematics (present value of $\lambda^{87} \mathrm{Rb}=1.42 \cdot 10^{-11} \mathrm{a}-1$ (Steiger and Jager, 1977)). If $D / N$ is such that eq. (8) gives $t_{a}=4.5 \mathrm{Ga}(D / N \sim 0.06599)$, assuming $c=-m / \lambda=1.25 \cdot 10^{-11} \mathrm{a}^{-1}$ (i.e. $\lambda_{0}=$ $\left.1.5 \cdot 10^{-11} \mathrm{a}^{-1}\right)$ we calculate a decrease of $\lambda$ per year $(m)$ of $-1.775 \cdot 10^{-22} \mathrm{a}^{-1}$, and a total decrease in 50 years of $8.875 \cdot 10^{-21} \mathrm{a}^{-1}$, which would be clearly invisible to our instruments. If $c=-m / \lambda=2.10 \cdot 10^{-11} \mathrm{a}^{-1}$ (Fig. $1 ; \lambda_{0}=$ $1.55 \cdot 10^{-11} \mathrm{a}^{-1}$ ), we calculate a decrease of $\lambda$ per year of $-2.982 \cdot 10^{-22} \mathrm{a}^{-1}$, with a total change in 50 years of $-1.491 \cdot 10^{-20} \mathrm{a}^{-1}$, also invisible to our instruments.

\subsection{The Apparent Age Coincide for Simultaneous Geological Events on Different Planets of a Planetary System}

Let us consider the case of geological events (for example formations of igneous rocks) which occur at the same instant in the geological time on different planets of the same planetary system. Let us assume to calculate the apparent age of these geological systems using the same parent-daughter $(P-D)$ systematics. Although $\lambda$, and consequently $m$, depend on the distance of the planetary matter from the star, ratio $m / \lambda$ does not depend on this distance. Therefore, if the same $P-D$ systematics is used, the apparent ages of events which occurred at the same instant in the geological time on different planets of a planetary syste, coincide.

Instead, even if the same $P$ - $D$ systematics is used to date the geological events, the apparent ages of systems which formed at the same instant in the geological time on planets of different planetary systems, generally differ. This, because the age value is related to ratio $m / \lambda$ - the ratio between the rate of change over time of the concentration of the physical species which determines the activation of the parent nuclide and the current concentration of this physical species in the surroundings of the respective stars - which is generally different.

\subsection{The Case of Different P-D Decay Systematics}

In the above discussion, we have considered the case of using the same $P$ - $D$ systematics to date the geological systems because it is possible, on principle, that different parent nuclides are activated by different physical species. Nevertheless, it is also possible that different nuclides are activated by the same physical species, i.e. that differences in the values of $\lambda$ are due to differences in the respective value of fraction $\varphi$. 
In this case, the apparent ages of a geological system calculated using those different $P-D$ systematics coincide, because ratio $m / \lambda$ has the same values in the two cases. However, if the values of apparent age obtained by different systematics coincide, it could be also possible that the physical species which activate the respective parent nuclides are different, but the respective $\lambda$ s did not change significantly over the true age of the system. In this case, the calculated apparent ages are (very) close to the true age of the system. This also occurs for young geological systems, even if $\lambda$ changed significantly since the beginning of the geological time (see Fig. 1 and eqs. (19) and (20)).

\section{Is the Age of the Earth and of Solar System $\sim 4.50 \mathrm{Ga}$ ?}

We have shown above that if it is assumed that $D / N$ is such that eq. (8) gives $t_{a}=4.50 \mathrm{Ga}$, and $c=-m / \lambda$ is in the range $1.2-2.1 \cdot 10^{-11} \mathrm{a}^{-1}$, the difference between the true age and the apparent age is $-120 \div-200 \mathrm{Ma}$. Now, we want to determine the range of values of parameter $c$ if $\lambda$ is assumed to decrease slowly and roughly gradually over the geological time, as we can suppose if the sun was not in perfect equilibrium.

Eqs. (19) and (20) give the apparent age of a geological system as a function of the true age, in the case, respectively, of linear and exponential decrease of $\lambda$ over geological time since the formation of the system. We re-arrange these two equations to obtain the true age as a function of the apparent age. In the case of linear decrease of $\lambda$ we have, from eq. (19):

$$
t=\frac{\lambda}{m}\left\{1-\sqrt{1-2\left(\frac{m}{\lambda}\right) t_{a}}\right\},
$$

and in the case of exponential decrease of $\lambda$, from eq. (20), we have:

$$
t=\frac{1}{c} \ln \left(1+c t_{a}\right) \text {. }
$$

Let us assume that $\lambda$ decreased slowly and in a roughly gradual way over geological time because the emission of energy from the sun was not perfectly stable (Fig. 2). The true $\lambda=\lambda(t)$ distribution is obviously unknown; however, we can consider the linear function of the time which best fits the points of the distribution (the dashed straight line in Fig. 2). We can also consider the exponential function which best fits the distribution (the continuous line in Fig. 2).

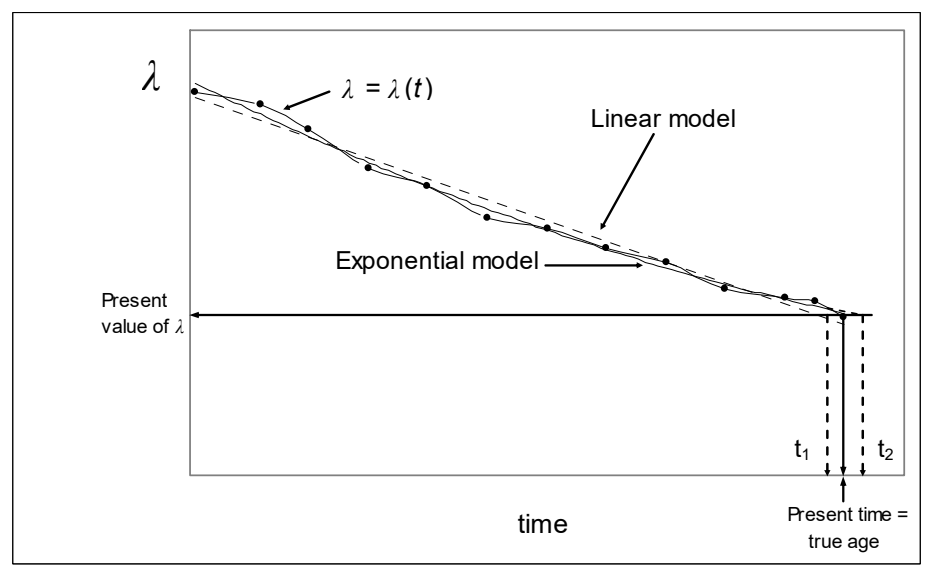

Figure 2. A possible $(t, \lambda)$ relationship in case the emission of energy from the sun decreased over geological time, and its linear and exponential best-fit models. 'Present time' is the true age of the system, and $t_{1}$ and $t_{2}$ are the true ages of the system as calculated by interpreting the distribution in terms of these two models, respectively. See text for further explanations

Note that we do not know the time-span elapsed since the formation of the sun and of the planetary objects; note, moreover, that the best-fit linear function intersects the time axis (the horizontal line which corresponds to the present value of $\lambda$ ) in a position which is generally different from the position of the intersection of the best-fit exponential function. We call the respective instants of intersection, which are also unknown, $t_{1}$ and $t_{2}$ (Fig. 2). We have: 


$$
t_{1}=\frac{\lambda}{m}\left\{1-\sqrt{1-2\left(\frac{m}{\lambda}\right) t_{a}}\right\}
$$

and

$$
t_{2}=\frac{1}{c} \ln \left(1+c t_{a}\right)
$$

If $\lambda$ decreased in a roughly gradual way, the $(t, \lambda)$ distribution is approximately fitted by a linear function of the time. In this case, the argument of the exponential function which best fits the distribution is small in absolute value, because, from a geometrical point of view, the exponential function is close to a linear function of the time as well. If it is so, the exponential equation (14):

$$
e^{c t}=\frac{\lambda_{0}}{\lambda}
$$

can be written as:

$$
1+c t \approx \frac{\lambda_{0}}{\lambda} .
$$

Eq. (25) shows that:

$$
c \approx \frac{\lambda_{0}-\lambda}{t} \frac{1}{\lambda}=-\frac{m}{\lambda}
$$

and substituting the relationship (26) into eq. (25) we obtain, for the best-fit exponential function:

$$
\lambda \approx \lambda_{0}+m t
$$

Therefore, if $\lambda$ decreased slowly and in a roughly gradual way over the whole geological time, parameter $c$ in the exponential model of $\lambda$ decrease is close to quantity $-m / \lambda$ in the linear model of $\lambda$ decrease.

Eq. (27), represents the 'linearization' of the best-fit exponential function. This 'linear' function is not perfectly equivalent to the best-fit linear function, because quantity $m / \lambda$ has not exactly the same value in eqs. (26) and (21), and also $\lambda_{0}$ is not exactly the same (Fig.2). However, the respective geometrical representations are certainly close to each other, so that $-m / \lambda$ in eq. (23) can be substituted by $c$ from eq. (26), resulting in very small deviations in the calculated value of $t_{1}$. In this case, eq. (23) gives the true age $t_{1}$ as eq. (24) gives the true age $t_{2}$, i.e. in terms of parameter $c$ and apparent age $t_{a}$ :

$$
t_{1} \approx-\frac{1}{c}\left(1-\sqrt{1+2 c t_{a}}\right)
$$

Since the two geometrical representations (of the best-fit linear function and of the best-fit exponential function) are close, the true ages $t_{1}$ and $t_{2}$ are also close to one another. Thus, if we assume a maximum value for the difference $t_{1}-t_{2}$ we can calculate the respective values of parameter $c$. We assume a limit value for this difference of approximately $10 \mathrm{Ma}$.

In eqs. (24) and (28), $t_{a}$ is the whole apparent geological time-span (4.5 Ga), and we calculate values of $t_{1}$ and $t_{2}$ assuming progressively higher values of $c$. If $c$ is in the range $1.0 \cdot 10^{-20}-1.0 \cdot 10^{-13} \mathrm{a}^{-1}$, the calculated true ages $t_{1}$ and $t_{2}$ are substantially undistinguishable from one another (the difference between $t_{1}$ and $t_{2}$ is always $<1 \mathrm{Ka}$ ), as well as from the apparent age (the deviation is $\leq \sim 1 \mathrm{Ma}$ ).

If $c=1.0 \cdot 10^{-12} \mathrm{a}^{-1}, t_{1}-t_{2}$ is $\sim 15 \mathrm{Ka}$, and if $c=1.0 \cdot 10^{-11} \mathrm{a}^{-1}$ this difference is $\sim 1.4 \mathrm{Ma}$, which is also negligible. The difference between $t_{1}$ and $t_{2}$ increases to about $10 \mathrm{Ma}$ if $c=3.0 \cdot 10^{-11} \mathrm{a}^{-1}$, and to approximately 24 and $68 \mathrm{Ma}$ if $c=$ $5.0 \cdot 10^{-11} \mathrm{a}^{-1}$ and $1.0 \cdot 10^{-10} \mathrm{a}^{-1}$, respectively (if $c>1.0 \cdot 10^{-10} \mathrm{a}^{-1}$ the difference between $t_{1}$ and $t_{2}$ increases rapidly). Thus $3.0 \cdot 10^{-11} \mathrm{a}^{-1}$ can be considered as the approximate upper limit of the range of 'possible' values of $c$. Fig. 3 shows the change of the values $t_{1}$ and $t_{2}$ as $c$ increases, and their progressive divergence. 


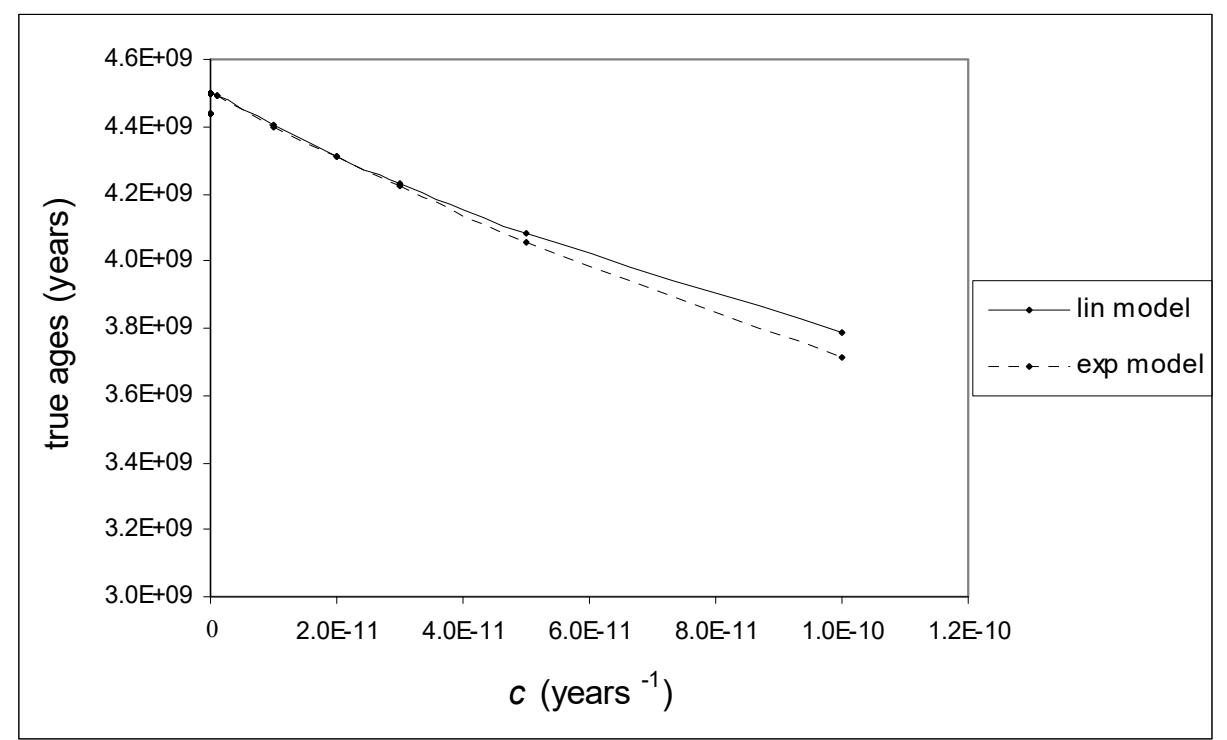

Figure 3. Relationship between parameter $c$ and true ages $t_{1}$ and $t_{2}$ calculated from linear and exponential best-fit of true $(t, \lambda)$ distribution, respectively, in the case $\lambda$ decreased slowly and in a roughly gradual way over the whole geological time

If $c$ is in the range $1.0 \cdot 10^{-20}-3.0 \cdot 10^{-11} \mathrm{a}^{-1}$, the true age is in the range $4.50-4.23 \mathrm{Ga}$. Therefore, in a scenario of slow and roughly gradual decrease of the emission of energy from the sun, the true age of the Earth and of the solar system could be significantly lower than $4.50 \mathrm{Ga}$, and as low as $4.23 \mathrm{Ga}$. In the case of ${ }^{87} \mathrm{Rb}^{87} \mathrm{Sr}$ dating systematics, the age value $4.23 \mathrm{Ga}$ corresponds to $\lambda_{0} \sim 1.60 \cdot 10^{-11} \mathrm{a}^{-1}$, so that, in this scenario, the average rate of change of $\lambda$ of ${ }^{87} \mathrm{Rb}$ would be $\sim 4.26 \cdot 10^{-22} \mathrm{a}^{-1}$ per year.

\section{Conclusions}

Arguments suggest that the process of radioactive decay is better re-interpreted in terms of second-order kinetics. Accordingly, the atomic systems of parent nuclides are not, as hitherto believed, 'radio-active', but 'radio-activated', and the rate of decay of each of these nuclides depends on the instantaneous concentration of one or more physical species which, by interacting with it, determines its transformation. The observed periodical variations in the value of $\lambda$ of alfa- and beta-emitting nuclides with the Earth-Sun distance, and the change in $\lambda$ value with the activity of the sun (Fischbach et al., 2009; Jenkins and Fischbach, 2009; Jenkins et al., 2009, 2010, 2012; Javorsek II et al., 2010; Sturrock et al., 2011) indicate that the process of radioactive decay cannot be longer interpreted in terms of first-order kinetics.

Since the value of $\lambda$ depends on solar activity, the age values calculated using parent-daughter decay systematics are probably apparent ones. If the Sun was not stable from the point of view of emission of energy, snd the value of parameter $\lambda$ slowly changed monotonically over the true age of a geological system, the calculated age value may significantly differ from the true age value, due to hyperbolic dependence of the calculated age on the value of the decay 'constant'. This in particular if the system is old.

If $\lambda$ decreased over the true age of a geological sample, the apparent time ran faster than the true time, and we can calculate the deviation of the apparent age from the true age in case $\lambda$ decreased according to possible models, for example a linear model and an exponential model. This deviation depends on the true age of the sample and on the relative rate of decrease of $\lambda$ (parameters $-m / \lambda=-\left(\lambda-\lambda_{0}\right) /(\lambda t)$ in the case of linear decrease, and $c=-d \lambda /(\lambda d t)$ in the case of exponential decrease).

The calculations also show that the apparent ages of coeval samples in different planets which orbit around the same star coincide, if the same $P-D$ decay systematics is used to date the samples. If different parent nuclides are activated by the same physical species, the apparent ages obtained by those radioactive $P-D$ systematics also coincide.

Instead, due to generally different changes over time in the emission of energy from the respective stars, the apparent ages of coeval systems in planets which orbit around different stars generally differ, even if calculated using the same $P$ - $D$ systematics. 
If the values of apparent age of a geological system determined using different $P-D$ decay systematics coincide, it is possible that the calculated ages are close to the true age of the system, or, alternatively, that the respective parent nuclides are activated by the same physical species.

If $\lambda$ decreases slowly and in a roughly gradual way over the true age of a geological sample, the linear function which best fits the true $(t, \lambda)$ distribution and the exponential function which best fits this distribution are close. In this case, we can write the equation which gives the true age of the sample as given by the linear model of decrease of $\lambda$ and the equation which gives the true age as given by the exponential model of decrease of $\lambda$ in terms of the same parameters, i.e. the apparent age of the sample and the parameter of exponential decrease of $\lambda, c$.

This permits to calculate the range of possible values of $c$, and, consequently, the range of possible values of the true age of the sample. If the geological sample is the sun-planets system (apparent age $\sim 4.5 \mathrm{Ga}$ ), we calculate $0<$ $c \leq 3.0 \cdot 10^{-11} \mathrm{a}^{-1}$, and the true age of the system in the range $4.5-4.23$

You may also consider the following issues:

- What is the theoretical, clinical, or practical significance of the outcomes, and what is the basis for these interpretations? If the findings are valid and replicable, what real-life psychological phenomena might be explained or modeled by the results? Are applications warranted on the basis of this research? (Note 1)

- What problems remain unresolved or arise anew because of these findings? The responses to these questions are the core of the contribution of your study and justify why readers both inside and outside your own specialty should attend to the findings. Your readers should receive clear, unambiguous, and direct answers. (Note 2)

\section{Acknowledgments}

This work was financially supported by C.N.R., Istituto di Geoscienze e Georisorse. The author is grateful to B. Zanettin, A. Della Giusta, A. Fasson and D. Roccato for discussions. Ida M. Samperi is very gratefully acknowledged for her generous care in language revision.

\section{References}

Alburger, D. E., Harbottle, G., \& Norton, E. F. (1986). Half-life of 32Si. Earth Planet. Sci. Lett. 78, 168-176.

Fischbach, E., Buncher, J., Gruenwald, J., Jenkins, J. H., Krause, D., Mattes, J., \& Newport, J. (2009). Time-dependent nuclear decay parameters: new evidence for new forces? Space Sci. Rev., 145, 285-335.

Javorsek II, D., Sturrock, P. A., Lasenby, R. N., Lasenby, A. N., Buncher, J. B., Fischbach, E., Gruenwald, J.T., ... Terry, B. (2010). Power spectrum analyses of nuclear decay rates. Astropart. Phys., 34, 173-178.

Jenkins, J. H., \& Fischbach, E. (2009). Perturbation of nuclear decay rates during the solar flare of 2006 December 13. Astropart. Phys., 31, 407-411.

Jenkins, J. H., Fischbach, E., Buncher, J. B., Gruenwald, J. T., Krause, D. E., \& Mattes, J. J. (2009). Evidence of correlations between nuclear decay rates and Earth-Sun distance. Astropart. Phys., 32, 42-46.

Jenkins, J. H., Herminghuysen, K. R., Blue, T. E., Fischbach, E., Javorsek II, D., Kauffman, A. C., Mundy, D. W., Sturrock, P. A., \& Talnagi, J. W. (2012). Additional experimental evidence for a solar influence on nuclear decay rates. Astropart. Phys., 37, 81-88.

Jenkins, J. H., Mundy, D. W., \& Fischbach, E. (2010). Analysis of environmental influences in nuclear half-life measurements exhibiting time-dependent decay rates. Nucl.Instr. and Meth. in Phys. Res. Sect. A, 620, 332-342.

Malatesta, L., \& Cenini, S. (1999). Principi di Chimica Generale. Con esercizi. Milano, Italy: Casa Editrice Ambrosiana.

Rieger, E., Share, G. H., Forrest, D. J., Kanbach, G., Reppin, C., \& Chupp, E. L. (1984). A 154-day periodicity in the occurrence of hard solar flares? Nature, 312, 623- 625.

Rutherford, E., Chadwich, J., \& Ellis, C. D. (1930). Radiation from radioactive substance. Cambridge, U.K: Cambridge university Press.

Siegert, H., Schrader, H., \& Schotzig, U. (1998). Half-life measurements of Europium radionuclides and the long-term stability of detectors. Appl. Radiat. Isot., 49, 1397- 1401.

Steiger, R. H., \& Jager, E. (1977). Subcommision on Geochronology: Convention on the use of decay constants in eo- and Cosmochronology. Earth Planet. Sci. Lett., 36, 359-362. 
Sturrock, P. A., Fischbach, E., \& Jenkins, J. H. (2011). Further evidence suggestive of a solar influence on nuclear decay rates. Solar Phys., 272 (1), 1-10.

\section{Copyrights}

Copyright for this article is retained by the author(s), with first publication rights granted to the journal.

This is an open-access article distributed under the terms and conditions of the Creative Commons Attribution license (http://creativecommons.org/licenses/by/4.0/). 